CARNETS OE Carnets de géographes

GÉOGRAPHES.

\title{
Une journée virtuelle sur un terrain virtuel avec une classe de seconde
}

\section{Anthony Merle}

\section{(2) OpenEdition \\ 1 Journals}

Édition électronique

URL : http://journals.openedition.org/cdg/2759

DOI : $10.4000 /$ cdg. 2759

ISSN : 2107-7266

Éditeur

UMR 245 - CESSMA

Référence électronique

Anthony Merle, « Une journée virtuelle sur un terrain virtuel avec une classe de seconde », Carnets de géographes [En ligne], 2 | 2011, mis en ligne le 02 mars 2011, consulté le 07 mai 2019. URL : http:// journals.openedition.org/cdg/2759; DOI : 10.4000/cdg.2759

La revue Carnets de géographes est mise à disposition selon les termes de la Licence Creative Commons Attribution - Pas d'Utilisation Commerciale - Pas de Modification 4.0 International. 


\title{
Une journée virtuelle sur un terrain virtuel avec une classe de seconde
}

\author{
ANTHONY MERLE \\ Agrégé de géographie \\ Enseignant dans l'académie de Grenoble \\ Chargé de cours à l'Université de Savoie
}

Le récit qui suit est inventé, même s'il se nourrit d'une expérience bien réelle. En effet, enseignant dans l'Académie de Grenoble, j'ai eu l'occasion de proposer à des élèves de seconde différentes séances menées sur Google Earth, en lien avec les différents chapitres de géographie alors au programme en 2009-2010. L'objectif premier était, par ce biais, de les initier à l'exercice cartographique ${ }^{l}$. Les questions et remarques formulées par certains élèves, ainsi qu'une réflexion sur la place de Google Earth dans nos démarches pédagogiques en géographie m'amène à proposer ce récit inventé, à l'écriture volontairement naïve, mais dont certains extraits sont pourtant véridiques.

Jeune enseignant, Joe Graphe n'est que stagiaire. Fraichement titulaire d'un concours dont le nombre de postes offerts diminue d'année en année, il pense que le plus dur a été réalisé. Mais voilà plus d'un mois qu'il enseigne et s'aperçoit bien que l'exercice n'est pas si simple. Il suit pourtant une formation deux jours par semaine dans un vieux bâtiment qui ressemble étrangement au château dans lequel s'égosillaient les candidats de la Star Academy. Cette ressemblance amuse d'ailleurs beaucoup ses collègues qui souvent comparent les principes du jeu télévisé à l'année qui les attend.

Après un mois de formation, Joe a déjà repéré ce qui était "à la mode » dans le milieu pédagogique: l'utilisation des T.I.C.E (Technologies de l'Information et de la Communication pour l'Education), la pédagogie différenciée, l'interdisciplinarité... Et puis il a toujours un ou deux collègues stagiaires pour lui rappeler qu'il faut "valider son $\mathrm{C} 2 \mathrm{i}$ (Certificat Informatique et Internet) »: Joe le sait bien, il a déjà jeté un œil sur les fameuses compétences à valider.

« Des séances en salle informatique, cela plaira forcément aux élèves » pense t-il tout en étant dubitatif sur les modalités de validation de ce certificat et sur son utilité réelle.

$* * *$

Joe Graphe est devant ses élèves de seconde, seize au total car ils sont en demi-groupe. Ceci permet d'avoir en moyenne un élève et demi par ordinateur, en comptant les éternelles machines en panne. Les élèves sont d'ores et déjà conquis par l'idée de faire de la géographie en salle informatique.

« Faute de les emmener sur le terrain, le terrain viendra à eux !» songe l'enseignant.

\footnotetext{
${ }^{1}$ Pour en savoir plus à ce sujet : MERLE Anthony, 2010, «Du globe virtuel à la production cartographique : utiliser Google Earth pour travailler les compétences liées à la cartographie en classe de seconde », Lettre de géomatique, en ligne sur http://eductice.inrp.fr/
} 
Une petite partie seulement du groupe connaît déjà Google Earth, bien souvent par une utilisation familiale. Le professeur a préparé un itinéraire à suivre sur le logiciel : il a placé des punaises virtuelles (icône " ajouter un repère »), et, lorsque l'élève clique sur celles-ci, des questions apparaissent. La réponse se trouve sur l'image satellite : l'élève apprend ainsi à décrypter l'image.

L'itinéraire à suivre se situe au sein de la Nouvelle-Orléans ${ }^{2}$, dans l'objectif de travailler sur la thématique des risques. Le titre de la séance est intitulé : "Katrina : une catastrophe uniquement naturelle? ». Le terrain est ici mobilisé non pas comme illustration du cours, mais véritablement comme moyen de construire le cours et la réflexion. L'objectif est de faire émerger chez l'élève la part de responsabilité des sociétés humaines et de leurs aménagements dans les catastrophes dites « naturelles ».

L'heure avance et les élèves sont totalement pris par la dimension ludique du logiciel, à tel point que Joe commence à douter de son efficacité pédagogique. En effet, avec cette histoire d'itinéraire et de punaises, il voit certains élèves s'empresser de répondre aux questions, parfois de façon bâclée, simplement parce que la curiosité les pousse à découvrir le plus rapidement possible la prochaine punaise. L'approche du terrain est donc résolument morcelée, discontinue : chacun va de lieu en lieu; en l'espace d'une seconde plusieurs dizaines de kilomètres sont parcourus et les élèves peinent à faire le lien entre ces lieux ainsi qu'entre les questions associées aux punaises. Ce n'est qu'à la fin du travail, lorsqu'une question de synthèse est énoncée ( « La catastrophe ayant touché la Nouvelle-Orléans en 2005 est-elle uniquement naturelle?») que le lien sera susceptible d'être réalisé.

Malgré tout, Joe sent que cette séance plaît aux élèves et qu'elle permet de nombreux apprentissages.

«Comment sur le terrain «matériel» les élèves pourraient-ils pratiquer aussi facilement le changement d'échelle? s'interroge-t-il. En effet, ils peuvent en quelques secondes localiser la Nouvelle-Orléans aux Etats-Unis, observer le trajet du cyclone au sein de l'espace Caraïbe, observer les dégâts du cyclone à l'échelle d'un quartier, voire d'une habitation. Le changement d'échelle ne peut être plus aisé ». Evidemment, l'enseignant aurait pu tenter une démarche similaire en classe à partir de photographies de paysages. Finalement, l'analyse de photographies n'est-ce pas non plus un moyen de «faire du terrain »? Que gagne t-on à se rendre sur Google Earth?

«Au moins avec Google Earth, les élèves peuvent dépasser ce que je leur donne à voir, songe l'enseignant. Ils sont libres de se déplacer ou de changer d'échelle et d'angle de vue! ». Mais finalement, le fait d'avoir placé des punaises avec questions/instructions, n'est-ce pas déjà trop les guider? «La prochaine fois, à eux de construire l'itinéraire et les punaises!», imagine $\mathrm{t}$-il.

Une élève l'interpelle :

«Monsieur! La question trois est impossible! Nous sommes en 2010, on ne peut donc pas voir les dégâts du cyclone puisque tout est réparé ! On ne peut pas répondre...»

Joe s'attendait à cette question. Il explique :

«Il existe une fonction sur le logiciel pour visualiser des images satellites passées. Cliquez ici ! Maintenant déplacez-vous sur l'échelle de temps et choisissez la date du cyclone - le 29 août $2005-$. C'est bon pour tout le monde ? »

Inutile d'attendre une réponse, il entend les élèves stupéfaits d'observer l'étendue des inondations. Là encore, sur le terrain « matériel », il n'aurait pas été possible d'opérer un tel

\footnotetext{
${ }^{2}$ La séance réellement proposée aux élèves est consultable sur le site de J-M Kiener : www.voyagesvirtuels.eu
} 
retour temporel : il aurait été nécessaire de chercher des traces de l'endommagement passé afin d'imaginer l'ampleur de la catastrophe.

«Monsieur! Comment ouvre-t-on Street View ! Moi, ça ne veut pas marcher !, s'exclame un élève sans lever la main ce qui a le don d'exaspérer l'enseignant.

- Tu as juste à cocher Street View dans la colonne de gauche ", répond le professeur, sûr que l'élève n'a même pas pris le temps de chercher un peu avant de l'interpeler.

Les élèves parcourent le terrain à travers les vues en $2 \mathrm{D}$ ou en $3 \mathrm{D}$, vues verticales plus ou moins zoomées, vues obliques, vues au sol via Street View (aujourd'hui automatiquement intégrée dans la version 6 de Google Earth) ou plus simplement grâce aux photos postées par les internautes. Si certains individus se repèrent dans l'espace davantage à partir de vues au sol, sorte de vision du quotidien, d'autres au contraire voient ou plutôt imaginent l'espace de façon aérienne, selon un point d'observation plus ou moins haut. L'avantage de Google Earth est donc de proposer différents angles de vision de l'espace: toutes ces perceptions différentes ne peuvent être que bénéfiques pour l'élève et sa capacité à se repérer dans l'espace. Là encore, il aurait été difficile d'envisager une telle palette de «visions de l'espace » sur le terrain « matériel ».

«Monsieur, j'ai fini ! Je fais quoi maintenant? interroge un élève en dessinant un petit cœur sur la feuille de sa voisine

- Moi aussi, surenchérit un autre

- Ils ont rarement été aussi efficaces, songe Joe. Peut-être aurais-je du ajouter deux ou trois punaises de plus... Et dire qu'il reste dix minutes avant la sonnerie.»

Finalement, l'enseignant décide de les laisser utiliser librement le logiciel pendant les dix minutes restantes.

« Voilà une bien drôle de consigne, se dit-il. Au moins ils se perfectionneront dans la maîtrise du logiciel. Et puis, ils sortiront de ces fameuses punaises... ». Il s'aperçoit que l'engouement des élèves pour cette nouvelle consigne est bien plus riche qu'il ne le pensait. Curieux, les élèves « volent» de lieu en lieu, parfois dans l'Ici (visualiser le lycée, sa maison...) parfois dans un Ailleurs plus ou moins empreint d'exotisme et d'altérité. Certains élèves se rendent sur leurs lieux de vacances, d'autres voguent vers des contrées qui les font rêver. Une élève tient par exemple à savoir si la ville de Miami est réellement telle qu'elle est présentée dans la série Les Experts. Ou quand le terrain virtuel sert de « contrepoids » à la virtualité télévisée... "Voilà peut-être de quoi aiguiser l'intérêt de certains pour la géographie » se satisfait l'enseignant.

« Monsieur! Je n'arrive pas à voir ma maison, s'étonne une élève.

- Est-ce que tu es sûre d'être au bon endroit? rétorque l'enseignant

- Oui, c'est bien là. Je reconnais le rond-point, mais il n'y a pas ma maison.

- Mais quand ta maison a-t-elle été construite ?

- Il y a huit mois que l'on habite dedans. Elle est toute neuve. »

Joe explique alors que les images satellites peuvent dater de plusieurs mois voire plusieurs années. En effet, voilà une limite de Google Earth, notamment en comparaison à une sortie sur le terrain «matériel » : l'espace n'est pas présenté en temps réel.

Déjà un autre élève interpelle l'enseignant :

« Monsieur! Venez voir!

- Oui

- Regardez, je suis en Corée du Nord 
- C'est bien, répond le professeur qui fait immédiatement le lien avec le devoir-maison donné la semaine précédente sur la fermeture des frontières nord-coréennes.

- Oui, mais j'ai beau chercher, je ne vois pas de missiles nucléaires. Y'a même pas de chars!»

Après une réponse décevant un élève qui pensait apercevoir Kim Jong Il une allumette dans une main un pétard nucléaire dans l'autre, Joe reste lui aussi perplexe. Finalement comment distinguer terrain «matériel » et terrain « virtuel »? Et pourquoi Google Earth ne pourrait-il pas remplacer définitivement les sorties sur le terrain « matériel»?

« Non, ce n'est pas possible, pense t-il. Pour commencer, Google Earth n'est pas un logiciel présentant l'espace en temps réel : il ne peut avoir la prétention de remplacer la pratique physique du terrain en géographie. Certes, les fonctions de Google Earth ne cessent de s'accroître, mais le terrain «matériel » reste marqué par une bien plus grande complexité apparente. Les jeux d'acteurs, la mobilisation des sens - odeurs, bruits, ambiances - sont absents ou presque sur le terrain virtuel. »

Il se rappelle alors cette phrase de M. Lussault relevant cette simplification qu'offre l'image par rapport au réel : "Les images proposées par Google Earth [...] exposent un espace en ordre, saisissable, compréhensible - tout en en faisant un spectacle qu'on peut regarder avec plaisir, justement parce que là l'espace fait bonne figure » (Lussault, 2007) Dans le même temps, n'est-ce pas cela qui fait l'intérêt pédagogique du logiciel ?

La sonnerie retentit. C'est la récréation et quasiment aucun élève n'a encore rangé ses affaires. Du jamais vu... Certains tardent même à sortir tant ils sont préoccupés par Google Moon (dont Joe n'a jamais mentionné l'existence...) et les différents toponymes qui jalonnent le satellite terrestre.

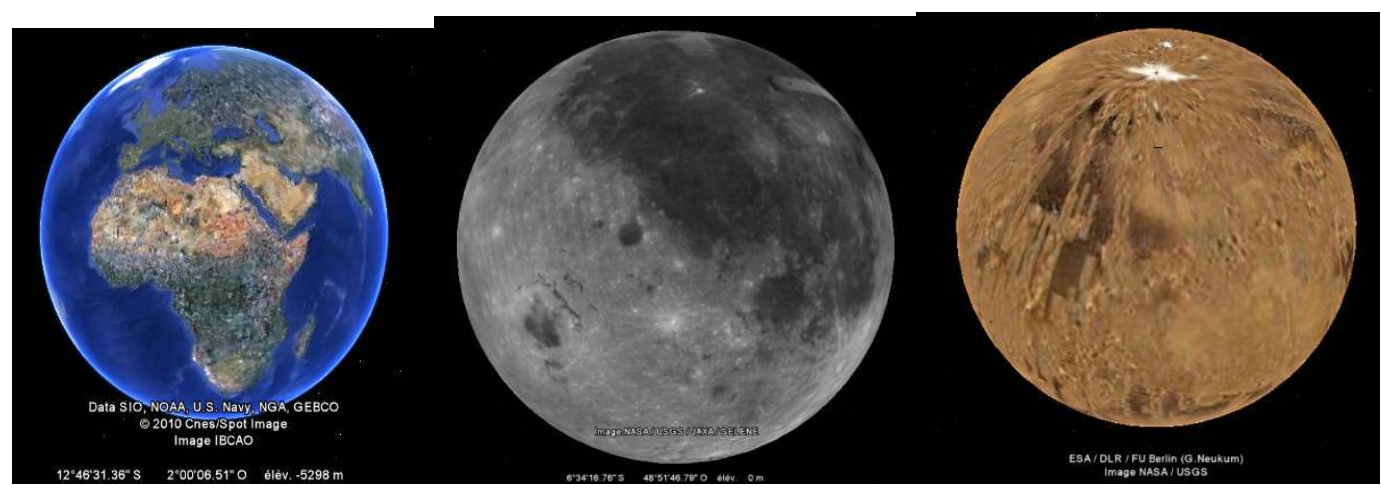

Visiter la Terre, la Lune, Mars...ou Google Earth et les limites de l'écoumène

Dix jours plus tard, Joe Graphe est en cours de psychologie cognitive de l'adolescent. Il repense aux copies corrigées la veille et dans lesquelles l'exemple de la Nouvelle-Orléans a servi d'illustration quasi-systématique au sujet proposé. Finalement, terrain virtuel ou terrain « matériel », la démarche géographique ne s'en trouve pas non plus bouleversée. Google Earth offre la possibilité pour le géographe de pratiquer le terrain d'une manière virtuelle, mais toujours afin de confronter ses modèles à la "réalité » géographique ou dans l'objectif de se servir de la « réalité » géographique pour faire émerger ses modèles. Comme le souligne 
Anne Volvey (2003) dans sa définition du terrain : "le terrain [est placé] soit en amont soit en aval de l'objet de recherche, les mouvements pouvant bien sûr se confondre ». Dans son cas, Joe Graphe avait placé le terrain de la Nouvelle-Orléans en amont de la réflexion des élèves. Evidemment, le choix du terrain était le sien, la Nouvelle-Orléans s'imposant alors de facto aux élèves. En quoi ce choix était-il pertinent? La pertinence du terrain tient-elle à son caractère exceptionnel ou à son éventuelle représentativité ? Finalement, pour l'enseignant, c'est tant l'utilisation pédagogique de l'étude de cas que la place et le statut du terrain dans la réflexion scientifique qui sont à questionner. A ce sujet, Y. Calbérac (2007) écrit d'ailleurs : «Le terrain est envisagé comme une instance de validation et de légitimation des savoirs géographiques. Cette légitimation relève du faire savoir, c'est-à-dire de la publicité qu'un chercheur fait de ses résultats et des moyens qu'il a mis en ouvre pour arriver à cette fin et pour gagner cette légitimité. Autour du faire savoir, l'instance de publicité et de légitimation, gravitent deux instances complémentaires : le savoir (l'énoncé géographique proprement dit, ce que le chercheur a mis en évidence) et le savoir-faire, c'est-à-dire la méthodologie mise en cuvre et qui garantit à la fois l'orthodoxie de la démarche et l'appartenance disciplinaire du chercheur. [...]. La pratique du terrain participe donc de la légitimation de la discipline. A l'inverse, en faire légitime le chercheur qui se conforme à ses canons méthodologiques ». Si le terrain semble faire partie d'une certaine doxa disciplinaire, quelle place pour le «terrain virtuel »? En d'autres termes, la communauté des géographes s'accorderait-elle à voir remplacer le mot « terrain » par « terrain virtuel» dans la citation de Y. Calbérac ?

Enfin, Google Earth n'est qu'une image de la réalité : ce n'est pas parce que l'on voit sur l'image une autoroute saturée que celle-ci constitue pour autant un axe majeur de transport. Les deux risques majeurs d'une survalorisation de l'imagerie satellite dans nos démarches pédagogiques seraient alors des généralisations hâtives et l'emploi systématique de l'image en guise de preuve irréfutable. M. Lussault (2007) reprenant les mots de R. Debray (1992) mentionne d'ailleurs les dangers de cette "équation de l'ère visuelle : le Visible $=$ le Réel = le Vrai ». Cependant, si cette équation est vraie pour le terrain « virtuel », elle l'est aussi pour le terrain « matériel »...

«La relation terrain virtuel / terrain matériel et réalité / irréalité s'avère donc bien peu pertinente » songe le jeune stagiaire.

Son voisin l'extrait alors de ses pensées :

« Et dire que l'on sera les derniers à profiter d'une année de formation.

- Oui, l'année prochaine ce ne sera pas évident pour les stagiaires et leurs élèves » chuchote Joe qui le pense sincèrement.

Pourtant, dans le même temps, le jeune enseignant peine à croire que les futurs stagiaires puissent regretter un jour de ne pas avoir suivi ce cours de psychologie cognitive...

\section{Bibliographie}

Calbérac Yann, 2007, "Le terrain des géographes, entre tradition et légitimation du chercheur" in Approches des terrains de recherches, Actes du colloque organisé par Doc'Géo, Bordeaux, 28 mars 2006, Cahiers ADES, n¹, mai 2007.

Debray Régis, 1992, Vie et mort de l'image, Paris, Gallimard, coll « Biblothèque des idées » Lussault Michel, 2007, L'homme spatial, Editions du Seuil.

Volvey A., 2003, «Terrain» in Lévy J., Lussault M., Dictionnaire de la géographie, de l'espace et des sociétés, Belin, pp.904-906. 
Carnets de géographes, $\mathrm{n}^{\circ} 2$, mars 2011 Rubrique Carnets de terrain 